\title{
Preliminary In-vivo Results For Spatially Coded Synthetic Transmit Aperture Ultrasound Based On Frequency Division
}

\author{
Gran, Fredrik; Hansen, Kristoffer Lindskov; Jensen, Jørgen Arendt; Nielsen, Michael Bachmann
}

Published in:

Proceedings of the IEEE Ultrasonics Symposium

Link to article, DOI:

10.1109/ULTSYM.2006.279

Publication date:

2006

Document Version

Publisher's PDF, also known as Version of record

Link back to DTU Orbit

Citation (APA):

Gran, F., Hansen, K. L., Jensen, J. A., \& Nielsen, M. B. (2006). Preliminary In-vivo Results For Spatially Coded Synthetic Transmit Aperture Ultrasound Based On Frequency Division. In Proceedings of the IEEE Ultrasonics Symposium (pp. 1087-1090). IEEE. https://doi.org/10.1109/ULTSYM.2006.279

\section{General rights}

Copyright and moral rights for the publications made accessible in the public portal are retained by the authors and/or other copyright owners and it is a condition of accessing publications that users recognise and abide by the legal requirements associated with these rights.

- Users may download and print one copy of any publication from the public portal for the purpose of private study or research.

- You may not further distribute the material or use it for any profit-making activity or commercial gain

- You may freely distribute the URL identifying the publication in the public portal 


\title{
Preliminary in-vivo results for spatially coded synthetic transmit aperture ultrasound based on frequency division
}

\author{
Fredrik Gran ${ }^{1}$, Kristoffer Lindskov Hansen ${ }^{2}$, Michael Bachmann Nielsen ${ }^{2}$ and Jørgen Arendt Jensen ${ }^{1}$ \\ 1) Center for Fast Ultrasound Imaging, Ørsted•DTU, Bldg. 348, \\ Technical University of Denmark, DK-2800 Kgs. Lyngby, Denmark \\ 2) University Hospital of Copenhagen, Rigshospitalet, Blegdamsvej 9, DK-2100 Copenhagen
}

\begin{abstract}
This paper investigates the possibility of using spatial coding based on frequency division for in-vivo synthetic transmit aperture (STA) ultrasound imaging. When using spatial encoding for STA, it is possible to use several transmitters simultaneously and separate the signals at the receiver. This increases the maximum transmit power compared to conventional STA, where only one transmitter can be active. The signal-tonoise-ratio can therefore be increased and better penetration can be obtained. For frequency division, the coding is achieved by designing a number of transmit waveforms with disjoint spectral support, spanning the passband of the ultrasound transducer. The signals can therefore be separated at the receiver using matched filtering. The method is tested using a commercial linear array transducer with a center frequency of $9 \mathrm{MHz}$ and $68 \%$ fractional bandwidth. In this paper, the transmit waveforms are designed as non-linear frequency modulated signals. This allows for efficient design of the amplitude spectrum of the signals. The duration of the signals was $25 \mu$ s and the bandwidth of each frequency band was $2.8 \mathrm{MHz}$. Eight frequency bands were designed which allowed for four transmitters to be active simultaneously. The method is compared to traditional STA with linear frequency modulation as means of temporal coding. The reference waveform was a $20 \mu$ s chirp at $9.37 \mathrm{MHz}$ with a bandwidth of $11.3 \mathrm{MHz}$. Penetration and resolution is evaluated using a tissue mimicking phantom. The increase in penetration for the frequency division method was approximately $2 \mathrm{~cm}$. The SNR was measured in the same type of phantom and an increase in SNR at depths between $3 \mathrm{~cm}$ and $10 \mathrm{~cm}$ of $7.2 \pm 3.6 \mathrm{~dB}$ was found. In-vivo experiments were carried out by an experienced sonographer. First, the common carotid artery was scanned on a 27 year old healthy male volunteer. The image quality was comparable for the two methods. To compare penetration depth of the two methods, the vesica fellea was scanned on the same volunteer. The frequency division method exhibited approximately $2 \mathrm{~cm}$ improvement in penetration compared to conventional STA.
\end{abstract}

\section{INTRODUCTION}

Spatio-temporal encoding has been suggested to improve signal-to-noise-ratio (SNR) in ultrasound systems [1]-[5]. The purpose of spatial encoding is to transmit acoustic waves from several spatial locations simultaneously, and separate the signals at the receiver. The transmitted power can thereby be increased which results in improved SNR without influencing the focusing capability of the system.

In [1] it was suggested to encode the different transmits using a Hadamard matrix. For a specific transmission, the excitation waveforms on the different transmitters are premultiplied with the elements of a row (or column) of a Hadamard matrix. In the next transmission, the next row (or column) is used as pre-multiplier for the waveforms. When all rows have been covered, and if the target geometry is assumed to be static, due to the orthogonal and binary nature of the Hadamard matrix, the decoding is reduced to simply adding and subtracting the different received signals. However, motion will generate artifacts due to incomplete cancellation of the different signals.

In [2] it was suggested to use orthogonal Golay code sequences as means of spatio-temporal encoding. The summed auto-correlation function of a set of Golay codes is a digital delta function. Two sets of Golay codes are said to be orthogonal if the summed cross-correlation is equal to zero for all lags. By letting one transmitter be represented by one set and another by the other orthogonal set, the intertransmitter interference can be totally canceled. Signal separation is achieved by simply correlating the received signals with the code sequences and summing the results. At the same time code compression is obtained due to the auto-correlation property of the Golay code. The approach assumes stationarity of the object between transmissions. Motion will result in not only incomplete separation of the different signals, but also degradation in the code compression.

In [3] spatial coding by frequency division (FD) was proposed. Here, a number of narrowband signals were designed, covering the entire passband of the transducer. The different transmitters are represented by signals with approximately disjoint spectral support. At the receiver, the signals are separated using matched filtering. To recover the maximum axial resolution of the system, data is acquired over a number of transmissions, so that the all frequency bands are covered, synthesizing broadband spectra for all transmitters. As for the other methods, the broadband synthesis will suffer from motion artifacts if the object under investigation is moving. A critical difference to orthogonal Golay coding is, however, that all inter-transmitter interference will be removed regardless of motion, since signal separation is obtained instantaneously at the receiver.

In [4], [5] the method was extended to also encompass temporal encoding in form of linear frequency modulation 
(LFM) and in [6] a simulation study was carried out to evaluate performance in synthetic transmit aperture (STA) blood flow estimation.

In this paper, the temporal coding is done using nonlinear frequency modulation. The narrowband waveforms are designed using the approach specified in [7]. The method described herein, offers flexibility for designing the amplitude spectrum which is crucial for creating a smooth synthesized spectrum for good sidelobe properties. Performance is evaluated experimentally using the RASMUS system [8] and invivo data are shown which were acquired by an experienced sonographer.

\section{METHODS AND MATERIALS}

\section{A. Equipment}

The experimental ultrasound system RASMUS [8] was used together with a BK8811 linear array transducer. The center frequency of this transducer is 9-12 MHz. For the transmitting aperture, virtual sources [9] were used. A virtual source consisted of 16 adjacent transducer elements focused behind the surface of the transducer. The focusing provided an F-number of 1.5. The transmission sequence and the focusing strategy can be found in [4].

\section{B. Spatio-temporal encoding}

Four virtual sources were used simultaneously. Therefore, eight narrowband waveforms were designed. The signals were divided into two overlapping sets [5]. The signals within a set had disjoint spectral support. The design method outlined in [7] were used to create the waveforms. The parameters for the waveforms were: bandwidth $2.8 \mathrm{MHz}$, duration $25 \mu \mathrm{s}$, and the desired amplitude spectrum was chosen to be the square-root of a Hanning function. The spectra for the matched filtered signals (which ideally should be Hanning functions) are given in the top plot in Fig. 1. To assure no interference between transmitters, the waveforms were divided into two sets where the waveforms within a set exhibited disjoint spectral support. The first set is given as the black curves in the top plot in Fig. 1 and the gray curves represent set number two. The bottom plot in Fig. 1 represent the synthesized broadband spectra (black curve) together with the transfer function of the transducer (gray curve).

\section{Reference method}

The reference method was based on STA using LFM as excitation waveform. The LFM had a bandwidth of 11.25 $\mathrm{MHz}$, a duration of $20 \mu \mathrm{s}$, center frequency $9.37 \mathrm{MHz}$ and a Tukey window was applied with $30 \%$ taper to reduce temporal sidelobes. The compression filter was the time reversed waveform multiplied by a Chebyshev window with $70 \mathrm{~dB}$ relative sidelobe attenuation.

\section{Beamforming}

The acquired data was beamformed using STA beamforming [10]. When the data from a specific virtual source was to be beamformed, the receiving aperture was chosen to be the 64 transducer elements centered around the virtual source. The receive apodization was a Hanning window.
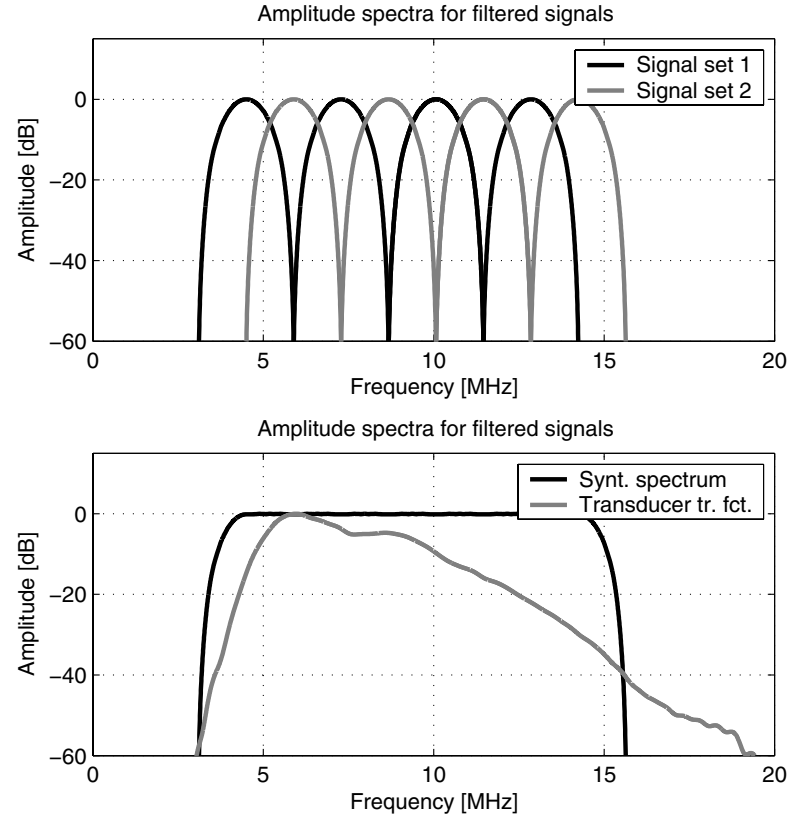

Fig. 1. The top figure shows the amplitude spectra of the eight filtered narrowband signals used to spatially encode the array. The spectra indicated in black represent code set one and the spectra indicated in gray represent code set two. The bottom figure shows the transfer function of the transducer in gray and the synthesized broadband spectra in black. The black curve is obtained by combining all eight filtered signals.

\section{EXPERIMENTAL RESULTS}

\section{A. Phantom measurements}

Three phantom measurements were carried out. The first experiment was aimed at evaluating depth of penetration and spatial resolution. A phantom from Dansk Fantomservice (Jyllinge, Denmark) was used with an attenuation of 0.5 $\mathrm{dB} /[\mathrm{cm} \mathrm{MHz}]$ at $20^{\circ} \mathrm{C}$. Inside the phantom, metal wires are suspended so that resolution and penetration can be analyzed. The B-mode images can be seen in Fig. 2. The LFM-STA method is given as the left image and the FD-STA method is given as the right image. The dynamic range was $40 \mathrm{~dB}$. The FD-STA method exhibited an increase in depth of penetration of $\sim 2 \mathrm{~cm}$.

The spatial resolutions (FWHM) for the string phantom experiment in Fig. 2 were calculated and the result is displayed in Fig. 3. The top plot shows the axial resolution and the bottom plot represents the lateral resolutions. The FD-STA method is shown in gray and the LFM-STA is shown in black. The LFM-STA displays slightly better lateral resolution compared to the FD-STA. The second experiment was carried out in a phantom from Dansk Fantomservice, model no: 571, with cysts inside the phantom. The aim of this experiment was to evaluate contrast. The result from this experiment is shown in Fig. 4, where LFM-STA is shown in the left image and FD-STA is shown in the right image. The dynamic range was $40 \mathrm{~dB}$. The speckle level inside the cyst at $30 \mathrm{~mm}$ compared to the speckle outside of the cyst was for LFM-STA -30.4 $\mathrm{dB}$ and for FD-STA $-29.6 \mathrm{~dB}$. The methods therefore exhibit comparable contrast. 

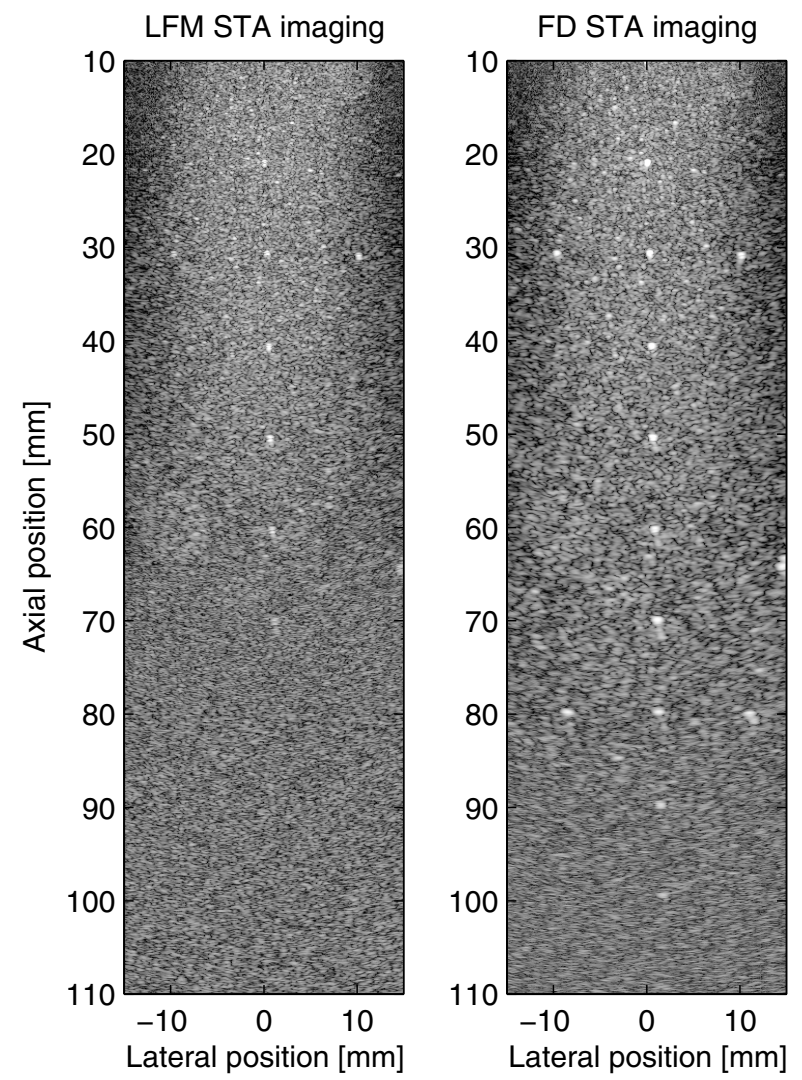

Fig. 2. The LFM-STA method is given as the left image and the FDSTA method is given as the right image. The dynamic range was 40 $\mathrm{dB}$. The FD-STA method exhibited an increase in depth of penetration of $\sim 2 \mathrm{~cm}$.

The last phantom experiment was carried out on the contrast phantom. However, the transducer was positioned so that only speckle was measured. The purpose was to calculate the gain in SNR for the FD-STA method compared to LFM-STA. Ten images were acquired with both the FD-STA method and the LFM-STA method. The images were averaged to estimate the noise-free image component. Thereafter, the SNR was calculated as a function of depth for the central part of the image. The result can be seen in Fig. 5. The gain in SNR over depth was $7.2 \pm 3.6 \mathrm{~dB}$.

\section{B. In-vivo measurements}

Two in-vivo scans were carried out by an experienced sonographer. The first image represents a cross-section of the common carotid artery of a 27 -year old healthy male. The two images are given in Fig. 6, where the left image is the LFM-STA method and the right image is the FD-STA image. The dynamic range of both images is $45 \mathrm{~dB}$. Post timegain-compensation (TGC) was applied to compensate for the attenuation. The second in-vivo image is taken on the vesica fellea (gall bladder) on the same patient. The purpose was to evaluate depth of penetration and contrast. The two images are presented in Fig. 7, where the left image is acquired with LFM-STA and the right with FD-STA. The dynamic range was again $45 \mathrm{~dB}$. The right image displays better penetration and the SNR in the cavity of the bladder is improved.
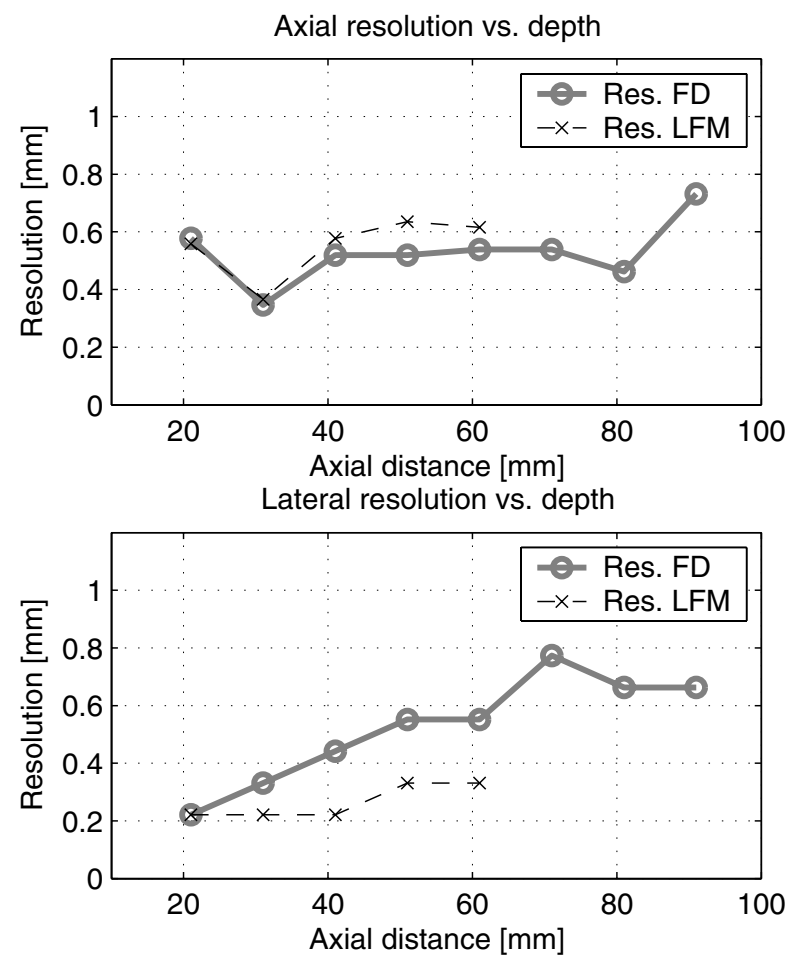

Fig. 3. The top plot shows the axial resolution and the bottom plot represents the lateral resolutions. The FD-STA method is shown in gray (and circles) and the LFM-STA is shown in black (and $\mathrm{x}$ ).
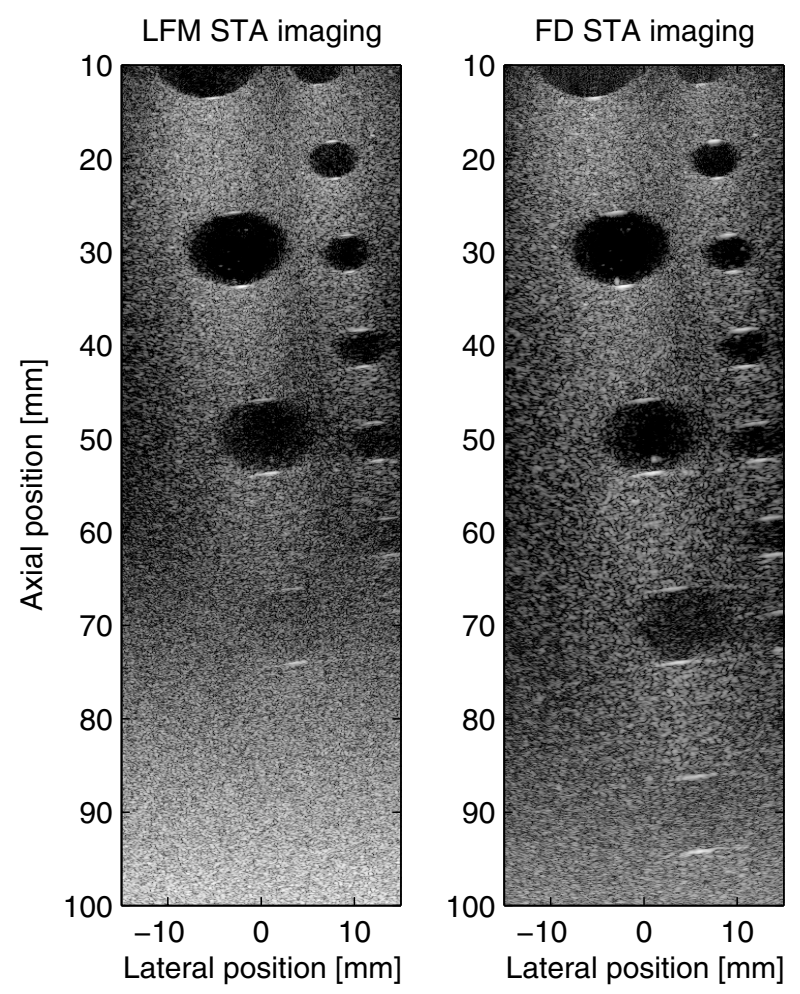

Fig. 4. Contrast experiment. LFM-STA is shown in the left image and FD-STA is shown in the right image. The dynamic range was 40 $\mathrm{dB}$

\section{CONCLUSION}

Spatio-temporal coding has been tested in-vivo. Also, a number of phantom experiments were carried out to assess 


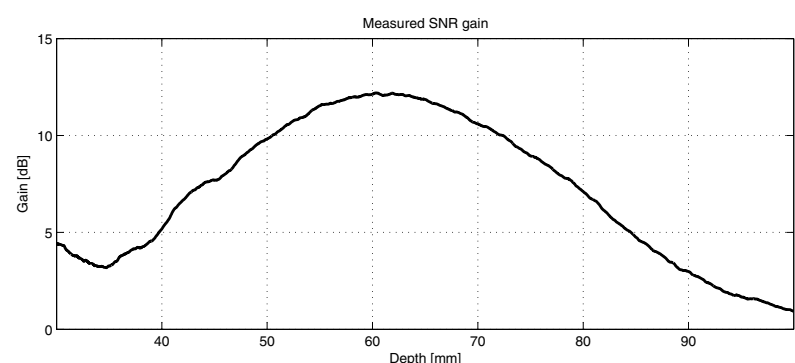

Fig. 5. The measured gain in SNR in $\mathrm{dB}$ for the FD method compared to LFM.
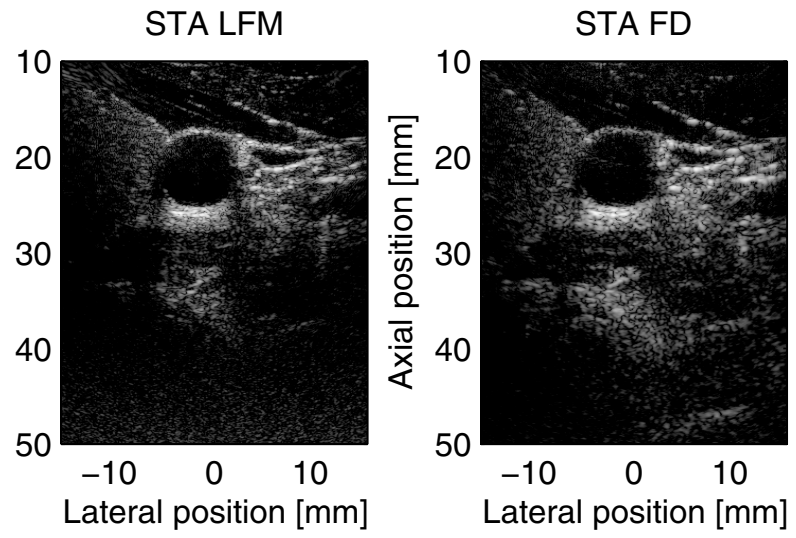

Fig. 6. The left image is the LFM-STA method and the right image is the FD-STA image. The dynamic range of both images is $45 \mathrm{~dB}$ Post TGC was applied to compensate for the attenuation.
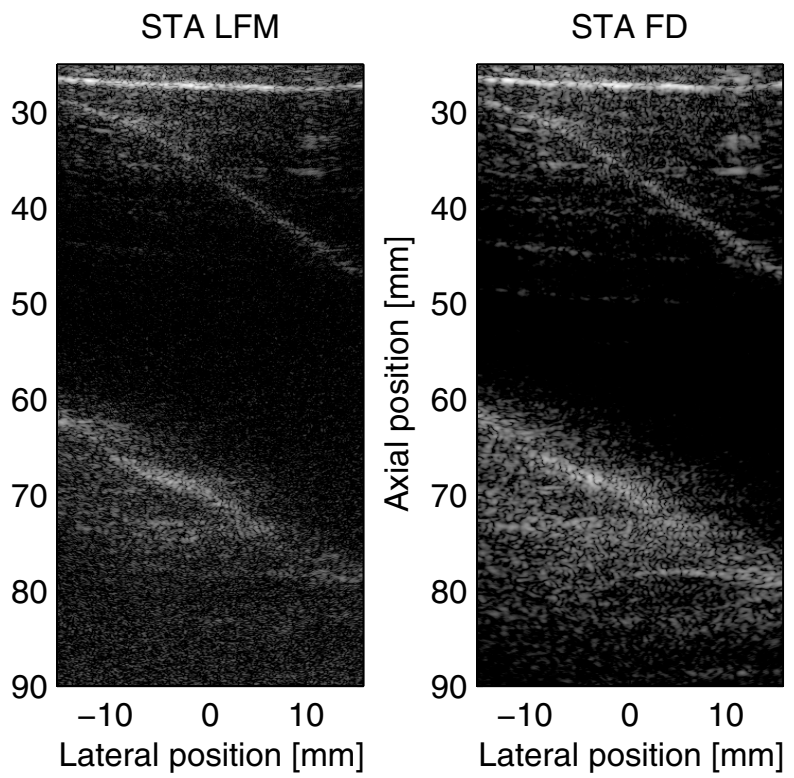

Fig. 7. The left image is acquired with LFM-STA and the right with FD-STA. The dynamic range was again $45 \mathrm{~dB}$. The right image displays better penetration and the SNR in the cavity of the bladder is improved.

performance in terms of spatial resolution, contrast and SNR. It was concluded that the FD-STA method provides the same image quality as LFM-STA. The depth of penetration was found to be increased significantly with the FD-STA method when compared to LFM-STA. Future work will be focused on making a larger clinical study, comparing the two methods. This study should include several different patients, different scans and a number of evaluating clinical experts.

\section{ACKNOWLedgment}

This work was supported by grant 274-05-0327 from the Danish Research Agency, the Radio-parts foundation and by B-K Medical A/S, Denmark.

\section{REFERENCES}

[1] R. Y. Chiao, L. J. Thomas, and S. D. Silverstein, "Sparse array imaging with spatially-encoded transmits," in Proc. IEEE Ultrason. Symp., 1997, pp. 1679-1682.

[2] R. Y. Chiao and L. J. Thomas, "Synthetic transmit aperture using orthogonal golay coded excitation," in Proc. IEEE Ultrason. Symp., 2000, pp. 1469-1472.

[3] F. Gran and J. A. Jensen, "Multi element synthetic aperture transmission using a frequency division approach," in Proc. IEEE Ultrason. Symp., 2003, pp. 1942-1946.

[4] F. Gran and J. A. Jensen, "Spatio-temporal encoding using narrow-band linearly frequency modulated signals in synthetic aperture ultrasound imaging," in Proc. SPIE - Progress in biomedical optics and imaging, 2005, vol. 5750 (1), pp. 405-416.

[5] F. Gran and J. A. Jensen, "Frequency division transmission and synthetic aperture reconstruction," IEEE Trans. Ultrason., Ferroelec., Freq. Contr., vol. 53 (5), pp. 900-911, 2006.

[6] F. Gran and J. A. Jensen, "Directional velocity estimation using a spatiotemporal encoding technique based on frequency division for synthetic transmit aperture ultrasound," IEEE Trans. Ultrason., Ferroelec., Freq. Contr., vol. 53 (7), pp. 1289-1299, 2006.

[7] F. Gran and J. A. Jensen, "Designing non-linear frequency modulated signals for ultrasound imaging," in Proc. IEEE Ultrason. Symp., 2006, p. Accepted for publication.

[8] J. A. Jensen, O. Holm, L. J. Jensen, H. Bendsen, S. I. Nikolov, B. G. Tomov, P. Munk, M. Hansen, K. Salomonsen, J. Hansen, K. Gormsen, H. M. Pedersen, and K. L. Gammelmark, "Ultrasound research scanner for real-time synthetic aperture image acquisition," IEEE Trans. Ultrason., Ferroelec., Freq. Contr., vol. 52 (5), May 2005.

[9] M. O’Donnell and L. J. Thomas, "Efficient synthetic aperture imaging from a circular aperture with possible application to catheter-based imaging," IEEE Trans. Ultrason., Ferroelec., Freq. Contr., vol. 39, pp. 366-380, 1992.

[10] S. I. Nikolov, Synthetic aperture tissue and flow ultrasound imaging, Ph.D. thesis, Ørsted•DTU, Technical University of Denmark, 2800, Lyngby, Denmark, 2001. 